\title{
Feasibility of mHealth intervention to improve uptake of antenatal and postnatal care services in peri-urban areas of Karachi: a qualitative exploratory study
}

\author{
Anam Feroz ${ }^{1}$, Narjis Rizvi ${ }^{1}$, Saleem Sayani ${ }^{2}$, Sarah Saleem ${ }^{1}$ \\ ${ }^{1}$ Aga Khan University, Community Health Sciences Department, Karachi, Pakistan; ${ }^{2}$ Aga Khan Development Network eHealth Resource Centre, \\ Karachi, Pakistan \\ Contributions: (I) Conception and design: A Feroz, S Saleem; (II) Administrative support: A Feroz; (III) Provision of study material or patients: A \\ Feroz; (IV) Collection and assembly of data: A Feroz; (V) Data analyses and interpretation: All authors; (VI) Manuscript writing: All authors; (VII) \\ Final approval of manuscript: All authors. \\ Correspondence to: Anam Feroz, BScN, MScHPM. Aga Khan University, Community Health Sciences Department, Stadium Road, PO Box 3500 , \\ Karachi 74800, Pakistan. Email: anam.feroz@aku.edu.
}

Background: In Pakistan, poor antenatal and postnatal coverage, has accounted for nearly $70 \%$ of preventable maternal deaths. Interventions implemented to improve uptake of antenatal and postnatal care (PNC) services have not shown significant improvements. Recent increase in cellphone penetration has brought forward mHealth as a potential strategy to enhance antenatal care (ANC) and PNC uptake. The objective of this study was to explore if mHealth technology is a feasible strategy to improve uptake of preventive maternal healthcare services in peri-urban areas of Karachi.

Methods: The study employed an exploratory qualitative research design using focus group discussions (FGDs) and key-informant interviews. FGDs were conducted with pregnant women, women in the postnatal period and lady health workers (LHWs) whereas key-informant interviews were conducted with maternal neonatal and child health experts and mHealth experts. The study data was analyzed using NVivo version 11. Results: This research found that women, healthcare providers and technology experts consider mHealth strategy has high potential to address barriers related to provision and utilization of ANC and PNC services. Healthcare providers and women understand the term mHealth and knew the benefits of mHealth services. Few of the women and healthcare providers are currently using mHealth for providing and receiving ANC and PNC services. Women and healthcare provider seemed ready for mHealth use however expressed challenges such as, illiteracy, cultural restrictions, lack of trustworthiness and misuse of technology.

Conclusions: This study informs that mHealth is operationally feasible, culturally acceptable and technologically appropriate strategy. For successful integration of mHealth technology in to existing maternal neonatal and child health service delivery structure, it is considered imperative to build a sustainable model of mHealth by involving government, local communities, telecommunication personnel, health care providers and mHealth and maternal neonatal and child health experts and through tailoring the design of the mHealth solutions in such a way that it benefits majority of women.

Keywords: Antenatal care (ANC); mHealth interventions; postnatal care (PNC); peri-urban areas of Karachi; qualitative study

Received: 23 October 2017; Accepted: 06 November 2017; Published: 08 November 2017.

doi: 10.21037/jhmhp.2017.10.02

View this article at: http://dx.doi.org/10.21037/jhmhp.2017.10.02 


\section{Introduction}

In Pakistan, despite reduction in the incidence of maternal deaths by $57 \%$ during 1990 to 2013 (1), the country [maternal mortality rate (MMR) of 276] is amongst the states having high MMRs (2). One out of 140 Pakistani women faces a lifetime risk of maternal death (3). Further reduction of MMR requires several maternal interventions including, antenatal care (ANC) and postnatal care (PNC) services. Literature suggests that these services can timely identify and manage preventable maternal morbidities such as Eclampsia, antepartum hemorrhage, obstructed labor, postpartum hemorrhage and puerperal sepsis which are contributing to nearly $70 \%$ of all maternal deaths (2).

Many interventions have been implemented in Pakistan to improve uptake of ANC and PNC services including capacity building of health care workers on key skills, provision of emergency obstetric care (EmOC) services, task shifting amongst healthcare workers, demand and supply side financial inducements. Despite all these efforts, only $37 \%$ of Pakistani pregnant women avail four ANC visits; the minimum number of ANC visits recommended by WHO. Moreover, $60 \%$ of women receive PNC services within the first 2 days following delivery (4).

The increasing penetration of cellphone over recent years has brought forward mHealth as a potential strategy that can enhance effectiveness and efficiency of healthcare delivery. 'Mobile health or mhealth refers to the use of mobile phones, personal digital assistants (PDAs), patient monitoring devices and other information and communication technologies (ICT) to support and deliver health and healthcare services' (5). According to the International Telecommunication Union 2015 figures, cell phone subscriptions have reached over 7 billion worldwide and in low/middle-income countries (LMICs) mobile penetration has reached over $90 \%$. Even in Pakistan the mobile penetration has now reached $76.6 \%$ with 139.9 million subscribers by mid-2015 (6). Research from LMICs demonstrates that mHealth technology has found to be useful for enhancing knowledge and increasing utilization of preventive maternal healthcare services by mothers (5-9).

Pakistan is currently deliberating on a plan to use mHealth technology for supporting community health workers (CHWs) (10). Within this scenario, it is imperative to understand the attitudes and perspective of health care workers' and Pakistani women about mHealth technology. This information will help in the development of technologically appropriate and culturally acceptable mHealth interventions. The current paper is based on a qualitative study that explored the views, experiences, and perceptions of public healthcare professionals and pregnant and postnatal women from the peri-urban areas of Karachi regarding the utilization of $\mathrm{mHealth}$ intervention for enhancing ANC and PNC service uptake.

\section{Methods}

\section{Study setting and study participants}

The study was conducted in two areas: Malir and the Aga Khan Hospital for Women and Children, Kharadar from July 2016 to September 2016. Malir is a semi-urban area having an estimated population of over 1 million. The postnatal women were interviewed from the Aga Khan Hospital for Women and Children, Kharadar as most women in Malir do not visit healthcare facilities to receive PNC services. Both these areas have diverse population in terms of ethnicity and socio-economic characteristics.

\section{Study design and procedures}

The study employed an exploratory qualitative research design using focus group discussions (FGDs) and key informant interviews (KIIs). FGDs and KIIs were conducted to explore the role of mHealth technology in improving uptake of ANC and PNC services. FGD participants were pregnant women, women in the postnatal period and lady health workers (LHWs) whereas KIIs were conducted with MNCH and mHealth experts. Semi-structured guidelines were used for FGDs and KIIs which included questions on knowledge about mHealth, current use of mobile phone for ANC and PNC services, women and healthcare provider readiness for mHealth use for ANC and PNC services, perceived benefits and challenges of mHealth use for ANC and PNC services, and proposed strategies to overcome anticipated challenges.

One of the eligibility criteria for the focus group was mobile phone ownership either by woman herself or by her household members. The FGDs and KIIs were continued till saturation of data (11). All the interviews were conducted after taking the informed consent of the participants. The ethical approval of the study was obtained from the Ethics Review Committee of Aga Khan University, Karachi, Pakistan. 


\section{Data analysis}

The data was transcribed from local to English language and was analyzed via qualitative data analysis software NVivo 11. Qualitative content analysis was conducted to understand the manifest content (what the transcript states) and latent content (understanding of the meaning of the transcript). Content analysis was carried out using software NVivo 11 in which transcripts were uploaded to offer easy and organized retrieval of data for analysis. Transcripts were read various times to develop an interpretation of the participants' perception of the role of mHealth technology in improving ANC and PNC service uptake, which constituted the unit of analysis. The text was divided into 'meaning units' which was shortened and labeled with a 'code' without losing the study context. Codes were then analyzed and assembled into categories to capture the manifest meaning. In the final step, sub-themes emerging from the main themes were developed. Two independent investigators performed the coding, category creation, and thematic analyses, and discrepancies were resolved to reduce researcher's bias. In the latent analysis, the emphasis was on determining the underlying meaning of the transcripts thus giving an account of how mHealth interventions could be integrated to improve ANC and PNC service uptake. To ensure the credibility of the research, study data were triangulated by the data source (pregnant women/women in the postnatal period, LHWs, MNCH experts and mHealth experts) and data methods (FGDs and KIIs), to compare alternative perspective and reveal any inconsistencies (12).

\section{Results}

A total of 46 participants were recruited in the study including pregnant women $(\mathrm{n}=18)$, women in the postnatal period $(n=12)$, LHWs $(n=12)$, MNCH experts $(n=2)$ and mHealth experts $(\mathrm{n}=2)$ (Table 1$)$.

Most of the FGD participants $(n=30)$ had personal mobile phone and only few had household phone. The majority of the FGD respondents $(n=38)$ owned basic mobile phones for sending and receiving calls. Very few of the FGD respondents $(n=19)$ were capable of sending and receiving messages through basic mobile phones. Only few FGD respondents $(n=4)$ owned smartphones and had access to WhatsApp, Facebook, Viber, and IMO. All the KIs, MNCH and mHealth experts, had smartphone and therefore had access to the internet and other applications such as WhatsApp, Facebook, Viber and IMO (Table 2).
Based on the data collected during FGDs and KIIs, six themes were identified. These included, (I) knowledge about mHealth for ANC and PNC services; (II) current use of mHealth for ANC and PNC services; (III) women and healthcare providers' readiness for using mHealth for ANC and PNC services; (IV) perceived benefits of mHealth use for ANC and PNC services; (V) perceived challenges of mHealth use for ANC and PNC services; and (VI) proposed strategies to overcome anticipated challenges.

\section{Knowledge about mHealth for ANC and PNC services}

The majority of the participants knew that mobile phone can be used for provision of ANC and PNC services, however, most of the respondents from FGDs (LHWs, pregnant women, and women in postnatal period) were not aware of the 'mHealth' terminology.

'Yes, I know the use of mobile phone for providing information to women about pregnancy care. However, I have not heard about this terminology (mHealth) before.' (A LHW in FGD).

Only a few of the illiterate respondents did not know that mobile phone can be used for providing or receiving healthcare services.

\section{Current use of mobile phones for ANC and PNC services}

On enquiry about the current uses of mobile phone, various purposes were mentioned by participants depending upon their requirements and context.

\section{Current use of mobile phone by experts and healthcare providers}

LHWs, MNCH and mHealth experts mentioned that mobile phones are currently being used for provider to provider communication, provider to patient communication, provider training and education, data collection, data management and for patient education and behavior change.

Experts mentioned that mHealth is used for enhancing communication between different cadres of healthcare providers. For example, LHW or a mid-wife gets expert advice from gynecologist while conducting high risk delivery.

Most MNCH and mHealth experts identified that mHealth provides guidance to patients through images, website links, inbound calls, outbound calls and messages. Experts further mentioned that mHealth plays crucial role for clarifying diagnosis to patients, enhancing their 
Table 1 Respondents characteristics

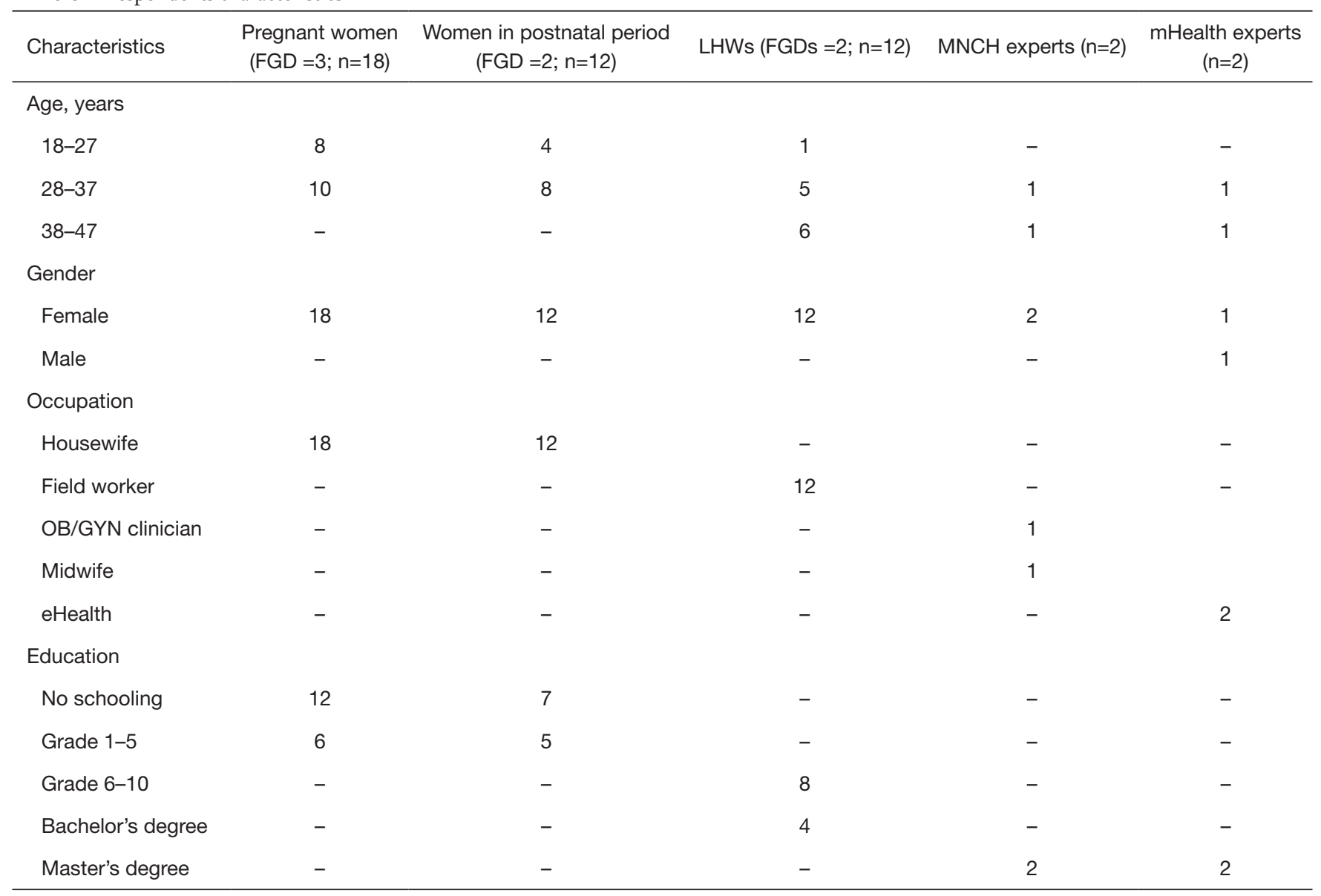

FGD, focus group discussion; LHWs, lady health workers; MNCH, maternal neonatal and child health; OB/GYN, obstetrician-gynecologist.

understanding, helping them in decision-making and building their confidence. Few LHWs reported that they provide their mobile numbers in their field sites so that women could resolve their queries through mobile phone communication without waiting for their next scheduled visit.

mHealth technology is used by MNCH and mHealth experts to train LHWs and other field-based staff on ANC and PNC services.

'We have used mobile phone technology to train community midwives in Gilgit-Baltistan areas. We have delivered training sessions through Skype calls and shared training materials in groups.' (A female MNCH expert in key-informant interview).

LHWs reported that they use mobile phones to complete data without even making home visits. One LHW mentioned that, when pregnant women move to her mother's house in the ninth month, they usually update their registers by getting information through mobile phones.

One mHealth expert reported that mobile phones have been used to update health information systems. According to the expert, community midwives were able to update information related to vital events (births, deaths, complications) on HIMS system by using mobile phones.

Majority of $\mathrm{MNCH}$ and mHealth experts mentioned that mHealth is used for patient education. Experts stated that through mobile phones, they share useful information materials (website links, images, text, and videos) with their routine clinic patients.

'I usually provide one website link (baby.com) to my patients for their pregnancy care. I believe that these links can better guide and motivate women about her nutrition and other aspects of ANC.' (A female MNCH expert in key-informant interview).

$\mathrm{MNCH}$ and mHealth experts mentioned that through mHealth they send important behavior change messages related to ANC and PNC to women, such as appointment 
Table 2 Technology experience of the respondents

\begin{tabular}{|c|c|c|c|c|c|}
\hline Technology experience & $\begin{array}{l}\text { Pregnant women } \\
(F G D=3 ; n=18)\end{array}$ & $\begin{array}{l}\text { Women in postnatal period } \\
\qquad(F G D=2 ; n=12)\end{array}$ & $\begin{array}{l}\text { LHWs (FGDs } \\
\qquad=2 ; n=12)\end{array}$ & $\begin{array}{l}\text { MNCH experts } \\
\qquad(n=2)\end{array}$ & $\begin{array}{l}\text { mHealth experts } \\
\qquad(n=2)\end{array}$ \\
\hline \multicolumn{6}{|l|}{ Mobile phone ownership } \\
\hline Personal phone access & 8 & 10 & 12 & 2 & 2 \\
\hline Household phone access & 10 & 2 & - & - & - \\
\hline \multicolumn{6}{|l|}{ Use of } \\
\hline Smartphone & - & 2 & 2 & 2 & 2 \\
\hline \multicolumn{6}{|c|}{ Able to send and receive messages } \\
\hline Yes & 3 & 4 & 12 & 2 & 2 \\
\hline No & - & - & - & - & - \\
\hline \multicolumn{6}{|c|}{ Able to use other mobile phone applications (e.g., WhatsApp, Viber, Facebook, etc.) } \\
\hline Yes & - & 2 & 2 & 2 & 2 \\
\hline No & 18 & 10 & 10 & - & - \\
\hline
\end{tabular}

FGD, focus group discussion; LHWs, lady health workers; MNCH, maternal neonatal and child health.

reminders, medication (supplementation) reminders, vaccination reminders and nutritional guidelines.

\section{Current use of mobile phone by pregnant women and women in postnatal period}

Pregnant and women in postnatal period mentioned that mobile phones are being used currently for patient to provider communication. Making phone calls to LHWs was mentioned as the primary function by pregnant women and women in postnatal period. The communication they have is informal and mostly related to arranging immunization appointments, receiving family planning supplies, inquiring about home visits and clarifying other health related queries.

\section{Women and healthcare providers' readiness for using mHealth for ANC and PNC services}

Readiness for using mHealth intervention was assessed in terms of women and healthcare providers' willingness to use mHealth and their future preferences with respect to mHealth modalities.

\section{Willingness to use mHealth in future}

Despite varying ability of respondents to use mobile technology, pregnant women and women in postnatal period were enthusiastic about potential mHealth applications and expressed their willingness to use mHealth services in future.

Pregnant women and women in postnatal period realize the value of mHealth for healthcare delivery. Women reported that through a mobile phone, their communication with lady heath workers would become much easier.

'I am very keen to know about this service. This would make our life much easier as we would not have to make frequent visits to bealth center to inquire short details on pregnancy care' ( $A$ pregnant woman in FGD).

mHealth and $\mathrm{MNCH}$ experts expressed dissatisfaction with the current state of healthcare and showed a willingness to adopt mHealth technology to improve existing service delivery structure. mHealth experts who used mobile phones for any healthcare service were considerably enthusiastic about the concept and were attracted by the speed and easy access. LHWs mentioned that using mobile phones they could contact medical expertise during an 
emergency and refer women to the equipped healthcare facility.

'Nowadays mobile phone technology is doing wonders. If we would use a mobile phone for providing ANC and PNC services, we could see a greater impact on maternal mortality and morbidity rates.' (A LHW in FGD).

Women preference with respect to mHealth modalities Text message sending and receiving were not commonly reported by pregnant women and women in postnatal period. Women reported that they usually find it difficult to read and understand text messages on mobile phone. Usually, they do not open text messages, and sometimes SMS messages are deleted as unread or deleted by other family members so they do not know what messages they are receiving. Very few women were able to send text messages to doctors and LHWs in Urdu language and in Roman Urdu (Urdu language written with the Roman script). Several language barriers to the use of SMS were described. These included the inability to read the English language in SMS and difficulty in using the English alphabet to write text messages. Most of the respondents preferred to receive and listen to audio messages.

Most of the LHWs were still in the basic stage of technological readiness. LHWs were able to send SMS in Urdu language and in Roman Urdu while some LHWs were comfortable in sending and receiving calls to resolve issues faster.

'I believe that sending mobile phone calls is more feasible as most of the women in our field are unable to read text messages due to illiteracy.' (A LHW in FGD).

When asked about what messages they would like to receive, pregnant women and women in the postnatal period mentioned medication reminders, appointment reminders and health education messages related to mother nutrition, child nutrition, childcare, danger signs in pregnancy and, normal signs of pregnancy, etc. Pregnant women mentioned that, these type of reminders and health education audio messages would help them self-track their progress over time and can ensure positive behaviors towards their health needs.

'Sometimes we are so busy in our daily routine that we forget the date of our ANC appointment and medication time. The reminder call would inform us about our ANC appointment and medication time.' (A pregnant woman in FGD).

A majority of women reported that SMS messages and calls should be sent in morning timings from 10:00 am to $01: 00 \mathrm{pm}$. Women further mentioned that receiving a call or message is most feasible in morning hours as their children and husbands are not at home and they could take time out to read a message and receive a call. Few women stated that messages and calls should be sent in evening hours from 05:00 pm to 07:00 pm. As few women who have household phone access could get these messages and calls at evening hours, when their husbands are back to home from work.

Most women reported that messages should be sent either in Urdu language or in Roman Urdu. Most of the women showed interest in receiving calls in the Urdu language. Only one woman reported that she would like to receive calls in the Sindhi language commonly used in the province.

\section{Healthcare provider preference with respect to mHealth modalities}

$\mathrm{MNCH}$ and mHealth experts suggested that messages and calls should be sent in evening hours in order to target the whole family as a unit. Moreover, they mentioned that this would allow them to receive these messages and calls as a couple. However, experts also suggested that to see the maximum effect, messages could be sent three times a day and Robocalls could be sent once a day. Moreover, two-way communication calls can be made fortnightly to resolve their concerns.

MNCH experts mentioned that the frequency of calls and messages would depend on the type of content, weeks of gestation for a pregnant woman and the duration of the postpartum period. Also, experts mentioned that messages could be sent to LHWs to raise their education about ANC and PNC services. As women are in direct link with LHWs, it would be good to create a primary link with LHWs in order to connect with community women. Experts further verbalized that the SMS and voice message content should be designed in a way that can address local myths and traditions around pregnancy.

\section{Perceived benefits of mHealth use for ANC and PNC services}

Respondents identified a number of benefits of mobile phone use for increasing uptake of ANC and PNC services including:

\section{Improved accessibility to healthcare providers}

In all FGDs, the ability to quickly communicate with providers in order to meet their health needs was an 
apparent sub-theme where mHealth held great promise.

'I believe that mobile phones would provide an easy access to healthcare providers. I could resolve my queries over the phone without even waiting for a long time in the clinic.' (A pregnant woman in FGD).

\section{Lesser cost than face-to-face consultations}

Respondents also mentioned that the cost of mobile phone consultation will be much less than the price of a face-toface consultation and found mHealth to be of good value. The majority of the respondents agreed that mHealth could be particularly helpful for the poor. Respondents verbalized that they could save their money through phone consultations and can utilize their savings to buy medicines and undergo diagnostic tests.

\section{Reduced travel cost and time}

Several respondents verbalized that mHealth could reduce transport costs and hassle of travel to access healthcare centers. Some respondents also saw mHealth as time-saving and an easier process to consult a doctor, especially when mothers are not sure of normal signs during pregnancy.

\section{Increased service utilization}

Most of the respondents mentioned that mobile phone communication would aid in increasing ANC and PNC service utilization. It will ensure better appointment adherence, increase in medication uptake, timely referral and improve responses to emergencies. Moreover, few LHWs verbalized that mobile phone communication can persuade pregnant women and women in the postnatal period to have timely checkups and assessments at antenatal and postnatal clinics. In addition, continuous follow-up could also be taken on mobile phone through messages and calls.

\section{Perceived challenges of mobile phone use for $A N C$ and PNC services}

Several respondents verbalized the perceived challenges of mobile phone use for ANC and PNC services.

\section{Illiteracy}

Literacy issues were pointed out as a consistent problem to provide mHealth services to peri-urban areas of Karachi. As most of the respondents couldn't read text messages, they usually delete the messages or left them unread. Sometimes they did not understand the meaning of the messages.
Participants expressed how illiteracy can be a barrier for mHealth use.

\section{Technological discomfort}

Few women reported that they have difficulty in operating smartphones and other commonly used mobile phones applications that include Facebook, WhatsApp, Viber, etc. In addition, most of the women verbalized that they are not competent in responding to messages which they receive on their basic mobile phones. Therefore, most of the women were only comfortable in receiving and making calls to their family members and LHWs. Few women reported that they usually send missed calls to their husbands and parents to receive a call from them at a later time.

\section{Cost of technology}

$\mathrm{mHealth}$ experts reported that lack of funding and increased cost of technology is a barrier to sustainability of the mHealth programs. $\mathrm{MNCH}$ and $\mathrm{mHealth}$ experts expressed their concerns that poor would not be able to use mHealth services that require access to smartphones. Moreover, they verbalized that if access to smartphones or basic mobile phones is ensured, they would be less able to use mHealth due to technological incapability and illiteracy. In addition, guaranteeing access to smartphones and the basic mobile phone is difficult, as it requires huge funding and resources.

\section{Lack of trust on technology}

A lack of trust in mHealth services was reported by few pregnant women and women in the postnatal period. They verbalized that they could not trust on information received through messages and calls unless they are sent by some recognized healthcare organization. Further women preferred to visit a doctor face to face for an initial consultation with follow-up via mobile phone.

'I will read messages only when it is sent by some authorized institution. I could not trust any message or call sent to me by any organization.' (A pregnant woman in FGD).

According to one pregnant woman, text messages can also be an insufficient source of information. The use of Internet and other smartphone applications were scarcely mentioned by the women.

\section{mHealth technology-not an absolute solution}

Few MNCH experts and LHWs felt that mHealth is not an absolute solution for all healthcare services related to ANC and PNC. They verbalized that mHealth is not appropriate for some services that require face to face interaction. 
$\mathrm{MNCH}$ experts verbalized that overreliance on technology will diminish the engagement with the community and the human value gained from live face-to-face consultation.

'I believe that we could not teach breastfeeding technique over the phone to first-time mothers. The counseling requires face to face interaction with mothers as they are very shy in communicating about breastfeeding their child.' (A LHW in FGD).

However, they verbalized that mHealth could be used as a supportive tool to compliment face-to-face consultations.

\section{Cultural restrictions}

Most women and LHWs reported that husbands and mothers-in-law would not allow women to receive calls and messages. The cultural restrictions still exist in peri-urban areas of Karachi where women are not allowed to have personal mobile phone access.

\section{Proposed strategies to overcome anticipated challenges}

Several respondents proposed strategies to overcome anticipated challenges of mHealth use for ANC and PNC services.

\section{Government involvement}

Government involvement was reported as a significant facilitator to overcome perceived barriers and sustain mHealth programs in future. Early involvement of government, policy makers and health management teams in planning and decision-making was considered to be essential to ensure the sustainability of mHealth program. $\mathrm{MNCH}$ and mHealth experts proposed working with the government at all stages from development to implementation. State involvement was also regarded as essential; to gain public trust in the intervention and setting guidelines, standards, and protocols for national implementation and adoption.

\section{Community involvement}

$\mathrm{MNCH}$ and mHealth experts highlighted the need for involving community leaders to raise awareness about mHealth services. Experts and LHWs mentioned that training the local population to run the program will overcome any potential obstacles related to acceptability and sustainability. Experts saw the community participation as a key to building trust and confidence in the program and put the population at ease. Consequently, they suggested the need to plan for a strong support team.

\section{Integration with existing services}

To overcome barriers to the acceptability of mHealth programs, $\mathrm{MNCH}$ and mHealth experts identified integration of mHealth technology in the existing service delivery package as a sub-theme. The integration subtheme, represent the idea that mHealth technology needs to connect with existing service delivery packages.

\section{Reduction of literacy and language barriers}

Most of the pregnant women and women in the postnatal period stated that SMS and audio messages should be provided in the Urdu language. However, only a few women verbalized that SMS and audio messages should be sent in the Sindhi language.

One way through which literacy barrier could be addressed would be by using audio messages. As the majority of the participants were able to send and receive calls through basic mobile phones.

\section{Coordination between key stakeholders}

Most MNCH and mHealth experts reported that successful delivery of mHealth interventions demands strong coordination between various key stakeholders to effectively deliver mHealth interventions. Moreover, one mHealth expert verbalized that the Ministry of Health can play a stewardship role in bringing together various stakeholders including government health officials, public health experts, $\mathrm{MNCH}$ experts, mHealth experts, telecommunication personnel, LHWs, women advocacy groups and community leaders to ensure effective planning and implementation of mHealth programs.

\section{Discussion}

The overarching goal of this study was to assess the feasibility of mHealth technology for enhancing ANC and PNC service uptake. This research informs that women, healthcare providers and technology experts feel that mHealth strategy has a high potential and is feasible for addressing barriers and hence increasing uptake of ANC and PNC services in peri-urban areas of Karachi. Most of the studies that have used mHealth technology to improve maternal health indicators were from developed countries (13-17) though, however one study from low resource settings has demonstrated use of mobile phones as influential tool for health-related communication (18). Keeping in view the facts that there has been high mobile phone penetration $(>76.6 \%)$ in the country $(6)$ and better 
understanding of benefits and use of mHealth services among most of the healthcare providers (LHWs, clinician, $\mathrm{MNCH}$ experts) and some of the healthcare recipients, mHealth technology seems to be a very feasible option. It is however important to note that for successful introduction of mHealth technology for enhancing ANC and PNC services, existing knowledge gap of mHealth among pregnant women and women in postnatal period needs to be addressed.

The study has informed that healthcare providers and women were currently using and expressed enthusiasm to use mHealth in future as they consider it to be more beneficial than face to face visits. It is important to note however that women mostly used call function to get health advice as they were not capable of sending and receiving text messages. Women suggested that mHealth can be used for reminders such as medication, appointment, followup visits and health education messages. Women and healthcare providers further informed that either of audio messages or two-way communication calls in Urdu and Sindhi language should be sent. In case text messages are to be sent then these should be in in Roman Urdu. In terms of time, evening hours were given preference. Studies from other countries have shown that information technology is a direct medium for both preventive and curative healthcare delivery and is specifically effective during emergency conditions $(18,19)$. In the context of Pakistan where around 60 percent of the population is residing in the rural areas far away from $\mathrm{MNCH}$ experts, mHealth would be an efficient and effective solution to address barriers of service delivery. However, since the majority of the women are illiterate, the design of the mHealth solutions need to be tailored in such a way that it benefits majority of women who owns simple mobile phones and women themselves can only comprehend audio messages or text messages in Roman English.

Study participants acknowledged that mHealth can increase uptake of ANC and PNC services through increasing accessibility to healthcare providers and reduction of consultation and transport cost, and waiting time. Study participants however felt that use of mHealth technology in healthcare delivery may encounter challenges. These challenges can be from healthcare providers' and clients' perspective. Clients' challenges could be illiteracy, cultural restrictions, technological discomfort, issues of trustworthiness and technology cost. Healthcare providers' challenges could be difficulties in management of complex health problems and misuse of technology.
Participants themselves identified solutions for many of these challenges. For instance, to overcome illiteracy and technology discomfort, text and audio messaging service were recommended. To manage complex health problems, mHealth end-users should be informed that some clinical conditions demands face-to-face interaction with doctors. To ensure trustworthiness in mHealth services, branding of messages can be done which was found useful in other studies $(20,21)$. In addition, community can be involved to build trust and confidence in the program. Regular reviews of the mHealth program and establishment of ICT policies have also found to ensure the quality of mHealth services (21). Moreover, to address issues related to cultural restrictions, several radio and mobile phone interventions (text and audio messages) have proven to be effective means of education and sensitization, particularly in reaching men, who are often the decision makers in their homes (22). To prevent misuse of technology, end-users of mHealth should be informed that mHealth cannot be an alternative for faceto-face visits and overreliance on technology will diminish the engagement with the community and the human value gained from live face to face consultation. To successfully deploy mHealth and achieve maximum uptake of ANC and PNC services, it is considered imperative to build a sustainable model of mHealth by involving government, local communities, telecommunication personnel, health care providers and mHealth and MNCH experts. Early involvement of government, policy makers and health management teams in planning and decision-making is considered to be essential to ensure the sustainability of mHealth program (22). If these perceived barriers are addressed in advance, integration of mHealth into existing practices might be more feasible and could increase buyin from the healthcare providers, technology experts and community women.

This was the first exploratory study that assessed the feasibility of mHealth as an intervention for improving uptake of ANC and PNC services in peri-urban areas of Karachi. The use of triangulation increased the credibility of research and reduced the effect of researcher's bias. Further, quantitative research should be conducted to quantify the findings of this study. Also, interventional studies could also be conducted to assess whether the benefits perceived translate into improved health outcomes and public health indicators. Some of the postnatal women were selected from the hospital as most women in Malir do not visit healthcare facilities to receive PNC services. However, the study could have identified and interviewed postnatal women from 
communities of Malir town as their perceptions might be different from those delivering at health facility.

The findings of this research demonstrate that mHealth has potential and is a feasible, culturally acceptable and technologically appropriate strategy to improve uptake of ANC and PNC services in peri-urban areas of Karachi. The study provides evidence that mHealth could evolve into a practice consortium to encourage community women to use ANC and PNC services in a timely and more efficient manner to improve existing service delivery structure. Issues such as preparing the community women, LHWs, other associated family members, discussing trust issues and providing authentic and reliable supportive services need to be taken into account before attempting to operationalize mHealth interventions. To overcome perceived barriers of mHealth use, we recommend integration of mHealth in existing service delivery packages by involving government, communities, telecommunication personnel, health care providers and mHealth experts.

\section{Acknowledgments}

The authors acknowledge the support of all administrators and Department of Health officials for the conduct of this study. They extend their gratitude to the study participants and the transcriber, for their contribution in this research.

Funding: None.

\section{Footnote}

Conflicts of Interest: All authors have completed the ICMJE uniform disclosure form (available at http://dx.doi. org/10.21037/jhmhp.2017.10.02).

Ethical Statement: The authors are accountable for all aspects of the work in ensuring that questions related to the accuracy or integrity of any part of the work are appropriately investigated and resolved. The study was conducted in accordance with the Declaration of Helsinki (as revised in 2013). The study was approved by the Ethical Review Committee of The Aga Khan University, Karachi, Pakistan (reference number: 4166-CHS-ERC-16). Each participant gave written informed consent.

Open Access Statement: This is an Open Access article distributed in accordance with the Creative Commons Attribution-NonCommercial-NoDerivs 4.0 International License (CC BY-NC-ND 4.0), which permits the non- commercial replication and distribution of the article with the strict proviso that no changes or edits are made and the original work is properly cited (including links to both the formal publication through the relevant DOI and the license). See: https://creativecommons.org/licenses/by-nc-nd/4.0/.

\section{References}

1. Estimates by WHO, UNICEF, UNFPA, The World Bank and the United Nations Population Division. Trends in Maternal Mortality: 1990 to 2013. 2014.

2. Pakistan Demographic and Health Survey 2006-07 Islamabad. National Institute of Population Studies Islamabad, Pakistan and Macro International Inc., Calverton, Maryland, USA, 2008.

3. Lifetime risk of maternal death (1 in: rate varies by country). 2015. Available online: http://www.indexmundi. com/facts/pakistan/indicator/SH.MMR.RISK

4. Pakistan - Demographic and Health Survey 2012-2013. Islamabad and Calverton, MA: National Institute of Population Studies and ICF International, 2013. Available online: http://www.nips.org.pk/abstract_files/PDHS\%20 Final\%20Report\%20as\%20of\%20Jan\%2022-2014.pdf

5. Nurmatov UB, Lee SH, Nwaru BI, et al. The effectiveness of mHealth interventions for maternal, newborn and child health in low- and middle-income countries: Protocol for a systematic review and meta-analysis. J Glob Health 2014;4:010407.

6. UCL PUBLIC POLICY. mHealth: Can mobile technology improve health in low- and middle-income countries? 2015.

7. Lund S, Nielsen BB, Hemed M, et al. Mobile phones improve antenatal care attendance in Zanzibar: a cluster randomized controlled trial. BMC Pregnancy Childbirth 2014;14:29.

8. Huq NL, Azmi AJ, Quaiyum MA, et al. Toll free mobile communication: overcoming barriers in maternal and neonatal emergencies in Rural Bangladesh. Reprod Health 2014;11:52.

9. Datta SS, Ranganathan P, Sivakumar KS. A study to assess the feasibility of Text Messaging Service in delivering maternal and child healthcare messages in a rural area of Tamil Nadu, India. Australas Med J 2014;7:175-80.

10. Lassi ZS, Kumar R, Bhutta ZA. Community-Based Care to Improve Maternal, Newborn, and Child Health. In: Black RE, Laxminarayan R, Temmerman M, Walker N, editors. SourceReproductive, Maternal, Newborn, and Child Health: Disease Control Priorities. Third Edition 
(Volume 2). Washington (DC): The International Bank for Reconstruction and Development/The World Bank, 2016, Chapter 14.

11. Guest G, Bunce A, Johnson L. How Many Interviews Are Enough?: An Experiment with Data Saturation and Variability. Field Methods 2006;18:59-82.

12. Patton MQ. Enhancing the quality and credibility of qualitative analysis. Health Serv Res 1999;34:1189-208.

13. Dalton JA, Rodger DL, Wilmore M, et al. "Who's afraid?": attitudes of midwives to the use of information and communication technologies (ICTs) for delivery of pregnancy-related health information. Women Birth 2014;27:168-73.

14. Jareethum R, Titapant V, Chantra T, et al. Satisfaction of healthy pregnant women receiving short message service via mobile phone for prenatal support: A randomized controlled trial. J Med Assoc Thai 2008;91:458-63.

15. Mackert M, Guadagno M, Donovan E, et al. Including men in prenatal health: the potential of e-health to improve birth outcomes. Telemed J E Health 2015;21:207-12.

16. Maslowsky J, Frost S, Hendrick CE, et al. Effects of postpartum mobile phone-based education on maternal and infant health in Ecuador. Int J Gynaecol Obstet 2016;134:93-8.

doi: $10.21037 /$ jhmhp.2017.10.02

Cite this article as: Feroz A, Rizvi N, Sayani S, Saleem S. Feasibility of mHealth intervention to improve uptake of antenatal and postnatal care services in peri-urban areas of Karachi: a qualitative exploratory study. J Hosp Manag Health Policy 2017;1:5.
17. Nguyen LT, Vu N, Duong HV, et al. mMOM - Improving maternal and child health for ethnic minority people in mountainous region of Thai Nguyen province of Vietnam through integration of mHealth in HMIS and userprovider interaction. Ann Glob Health 2015;81:195-6.

18. Andreatta P, Debpuur D, Danquah A, et al. Using cell phones to collect postpartum hemorrhage outcome data in rural Ghana. Int J Gynaecol Obstet 2011;113:148-51.

19. Bachiri M, Idri A, Fernández-Alemán JL, et al. Mobile personal health records for pregnancy monitoring functionalities: Analysis and potential. Comput Methods Programs Biomed 2016;134:121-35.

20. Thondoo M, Strachan DL, Nakirunda M, et al. Potential roles of Mhealth for community health workers: formative research with end users in Uganda and Mozambique. JMIR Mhealth Uhealth 2015;3:e76.

21. Chandra PS, Sowmya HR, Mehrotra S, et al. 'SMS' for mental health-feasibility and acceptability of using text messages for mental health promotion among young women from urban low income settings in India. Asian J Psychiatr 2014;11:59-64.

22. Battle JD, Farrow L, Tibaijuka J, et al. mHealth for Safer Deliveries: A mixed methods evaluation of the effect of an integrated mobile health intervention on maternal care utilization. Healthc (Amst) 2015;3:180-4. 